\title{
In-Situ Monitoring of Layer-Wise Fabrication by Electrical Resistance Measurements in 3D Printing
}

\author{
Alexander Dijkshoorn, Patrick Neuvel, Stefano Stramigioli, Gijs Krijnen \\ Robotics and Mechatronics, University of Twente, Enschede, The Netherlands \\ Email: a.p.dijkshoorn@utwente.nl
}

\begin{abstract}
This paper introduces a characterization technique to study 3D-printing of conductors and sensors during fabrication. Currently characterization of 3D-printed sensors is done after fabrication. In our novel method, however, the electrical resistance is monitored in-situ by electrically contacting the part in the beginning of the print process. This way, the effect of every additional layer on the total resistance is determined. Our new experimental method opens up ways to study 3Dprinting of sensors in order to better understand the processes at hand, e.g. it may allow distinguishing between bulk and interlayer resistances. FEM simulations and experiments are used to validate the use of this new method.
\end{abstract}

Index Terms-Electrical Resistance, Monitoring, 3D-Printing, Fused Deposition Modelling

\section{INTRODUCTION}

3D-printing of conductors by means of Fused Deposition Modelling is a promising technique for fabrication of customisable conductors and sensors [1], [2], [3]. FDM works by means of extruding molten plastic line per line, one layer at a time. Usually conductive-polymer composites are used to obtain electrical conduction with FDM, e.g. a polymer filled with nano-particles like carbon black or carbon nanotubes [4], [5], [6]. The layer-wise fabrication process introduces anisotropic electrical properties [7], [8], by improper fusion between layers and between lines or track-elements (traxels). These anisotropic properties can affect or even improve sensor performance [9], [10]. Currently the (anisotropic) electrical properties of 3D-printed conductors and sensors are characterized after the fabrication process. This is mainly done with global resistance or impedance measurements [8], [11], although a recent study also shows Scanning Electron Microscopy and infrared thermography measurements to determine the distributed electrical properties on single layers [12]. With these methods it is very difficult to distinguish the effect of the printing parameters on the electrical resistance of the individual layers in a larger print. In-situ measurements during printing can offer a solution in this respect. Several insitu techniques already exist for quality monitoring in FDM printing. Techniques are, among others, based on optical scanning [13], computer vision [14], acoustics [15], vibrations [16], strain [17], rheological [18] and thermal measurements [19]. However, to the best of the author's knowledge an in-situ

This work was developed within the PortWings project, funded by the European Research Council, Grant Agreement No. 787675. and the Wearable Robotics programme, funded by the Dutch Research Council (NWO). technique for monitoring the electrical properties has not yet been proposed. Therefore this paper presents the first in-situ monitoring technique of the electrical resistance in FDM. By means of inserting electrical contacts before or during printing (e.g. the electrical connections of a conductive sensor) the reduction in resistance by every added layer on top of the electrical contacts can be measured and the effects of printing parameters can be studied.

The following sections present the measurement principle and show experimental data and FEM simulations to proof the use of this technique for monitoring 3D-printing of sensors.
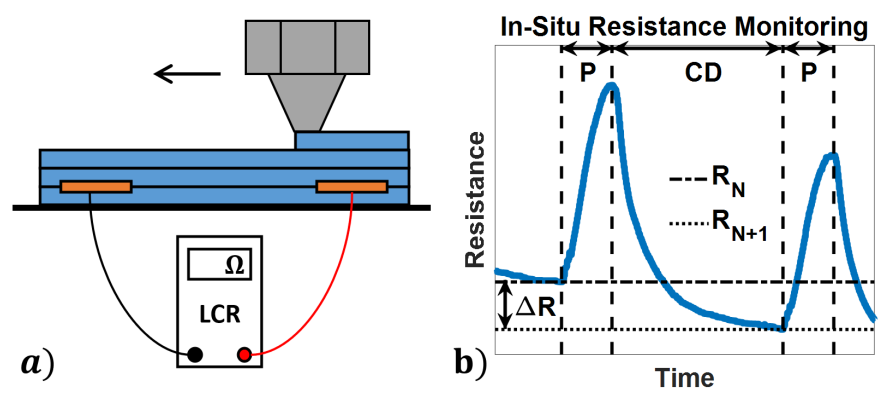

Fig. 1. a. Electrical contacts (e.g. used as connections to the printed sensor) are stuck onto the printing bed or embedded inside the initial layers of the 3D-print and used to monitor the electrical resistance. A 3D-printing nozzle extrudes molten plastic onto the sample layer by layer. The electrical resistance can be measured with a multi- or LCR- meter during printing. Ideally every added layer aids in lowering the electrical resistance. b. During printing $(\mathrm{P})$ the temperature dependent resistance goes up. After printing the resistance goes down during cool down (CD). The added layer yields a lower total resistance (going from $N$ to $N+1$ layers), which is followed by printing another layer.

\section{MeAsuREMENT PRINCIPLES}

The measurement principle is based on a basic electrical resistance measurement. Electrical contacts are fixed on the printing bed or embedded during printing to monitor the electrical resistance (and may later be used as electrical contacts for the sensor), figure 1.a. This can be used to study the changes in electrical resistance with added layers, the contact resistance between layers and the effect of printing parameters. The method can also be applied as a proxy to monitor other properties of parts that use conductive-polymer composites, for example for the mechanical, thermal or electrostatic charge dissipative nature of these materials [20]. One challenge for this measurement principle is the strong rise in electrical resistivity due to an increase in temperature (the targeted 
materials generally have a positive temperature coefficient, or PTC), often followed by a negative dependence of the resistivity on the temperature above the melting temperature [21]. The general accepted explanation for the PTC effect is believed to be a mismatch of thermal expansion coefficient between polymer and filler, especially at a phase transition (melting) of the polymer matrix [21]. This is encountered with FDM when the hot plastic and nozzle heat up the sample, causing the electrical resistance to increase during printing, phase $\mathrm{P}$ in figure 1.b. This challenge can be reduced or overcome by letting the sample cool down between printing of each layer, phase $\mathrm{CD}$ in figure 1.b. Hence, during printing of a layer the resistance ramps up and after printing the resistance drops exponentially.

\section{Methodology}

\section{A. Experimental Set-Up}

Experiments are performed with a customised Ultimaker 2 printer with a BondTec direct drive extruder, a water cooled hot end and an E3D stainless steel nozzle of $0.8 \mathrm{~mm}$. Samples are designed in SolidWorks CAD software and slicing is performed with Cura. As materials a conductive carbon black filled Polylactic Acid (PLA) called Proto-Pasta [22]) and a conductive carbon black filled TPU filament called PIETPU 85-700+ from Palmiga Innovation [23] are used. The samples are plates of $40 \mathrm{~mm}$ by $40 \mathrm{~mm}$ with a layer thickness of $250 \mu \mathrm{m}$, where they have a different number of layers depending on the test. Copper tape of $6.35 \mathrm{~mm}$ wide and $66 \mu \mathrm{m}$ thick with conductive acrylic adhesive is used as electrical contacts. Alignment tabs are printed on the side of the sample to place two pieces of copper tape consistently at a distance of $16 \mathrm{~mm}$, figure 2.a. The tape is added after printing two layers of material for reliable embedding. 6 small holes are punched in the tape and touched with a soldering iron at $400{ }^{\circ} \mathrm{C}$ to prevent the printer pushing the tape away when printing. The print bed is kept at $50{ }^{\circ} \mathrm{C}$, whereas the nozzle is kept at $230^{\circ} \mathrm{C}$. The fan is not used to reduce cooling below the print bed temperature. Per sample a single infill angle is used of either $0^{\circ}$ or $90^{\circ}$, as shown in figure 2.a. The extrusion multiplier (or flow rate) is taken at $100 \%$ or $110 \%$. Electrical resistance measurements are performed with the DC mode of an LCR (UNI-T UT612 LCR Meter). After printing each layer, the printer is paused for 5 minutes to let the sample cool down before printing the next layer.

\section{B. FEM Simulations}

In order to test our understanding of the measurement method, FEM simulations are performed (the inter-layer resistance complicates analytical calculations). The structures are simulated in 2D using the Electric Currents module in COMSOL. A 2D simulation can be used, since the sample has a uniform cross section. A voltage terminal and a ground are included to represent the copper electrical contacts over which the resistance is measured. Resistance between the printed layers is implemented through the contact impedance functionality. A parameter sweep is performed over the number of layers (ranging from 2 to 12, where the electrical contacts are placed on top of the second layer), for resistivity $(\rho)$ values of $0.15 \Omega \mathrm{m}$ and $0.25 \Omega \mathrm{m}$, and for isotropic conditions (a sample without inter-layer resistance) and an inter-layer contact resistance $(\sigma)$ of $1 \times 10^{-2} \Omega \mathrm{m}^{2}$.

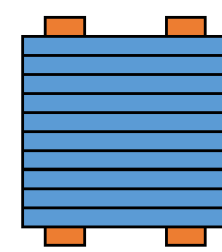

$90^{\circ}$ Infill

a)
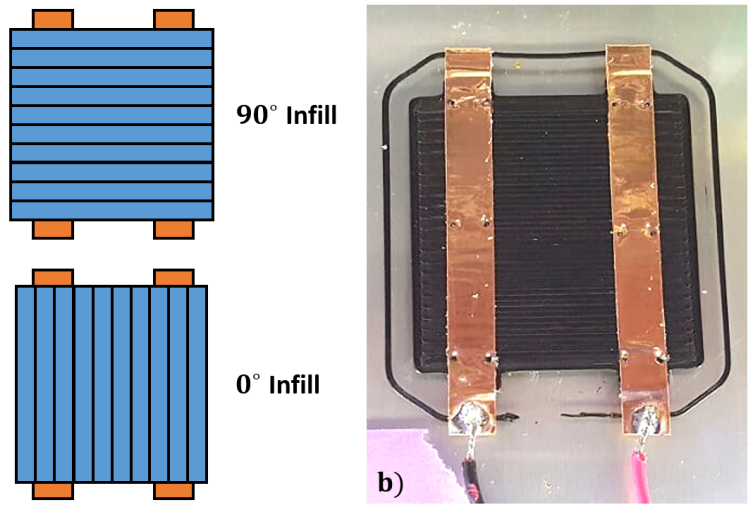

Fig. 2. a. The two infill angles used in the experiments: printing parallel $\left(0^{\circ}\right)$ and perpendicular $\left(90^{\circ}\right)$ to the electrical contacts. b. The first two layers of a sample with electrical contacts added on top. The electrical contacts are punctured with six holes each and touched with a soldering iron to fixate them.

\section{RESULTS}

The first two layers of a sample with the copper contacts are shown in figure 2.b. The infill in this sample is $90^{\circ}$, yielding traxels perpendicular to the electrical contacts. The resistance measurement is started directly following the application of the electrical contacts.

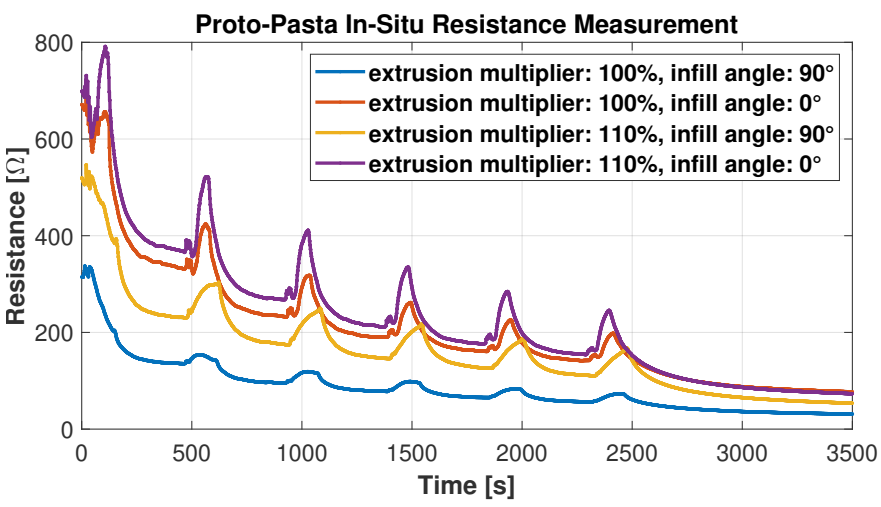

Fig. 3. Resistance over time for various print settings (extrusion multiplier, infill pattern) for conductive PLA. The peaks arise from heating during and subsequent cooling down after printing, as illustrated in figure 1.b.

Figure 3 shows measurements for various Proto-Pasta samples, whereas figure 4 shows them for ETPU samples. The printing of successive layers can be identified from the periodic peaks. Furthermore it becomes clear that every added layer lowers the total resistance. The resistance starts high and drops before the first layer is printed, since adding the electrical contacts with the soldering iron heats up the material significantly. Next to that the measurements show that an extrusion multiplier of $100 \%$ yields a lower resistance than $110 \%$ and 
an infill angle of $90^{\circ}$ yields a lower resistance than $0^{\circ}$ for both materials (even as measured after several days). Moreover for infill angles of $90^{\circ}$ the peak of the first layer is very small compared to the other peaks. Finally the peaks for $0^{\circ}$ are always sharper and more pronounced than for $90^{\circ}$.

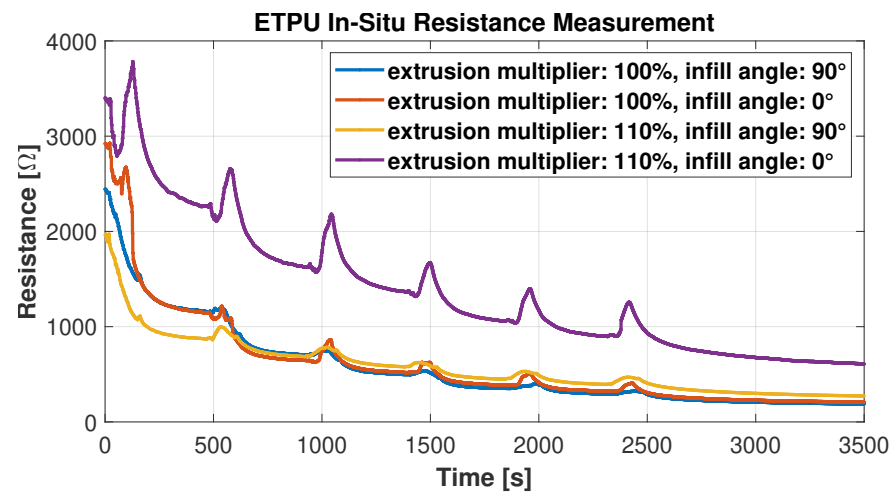

Fig. 4. As Figure 3 but results are for conductive TPU.

Additional measurements are performed on a Proto-Pasta sample composed of 12 layers for comparison with the FEM simulations. It is fabricated with an extrusion multiplier of $100 \%$, an infill angle of $0^{\circ}$, an increased cool down period of 7 minutes and copper contacts on top of the second layer. Figure 5 compares the measured total resistance versus the number of layers with the corresponding FEM simulations for an isotropic model and for a model with high interlayer resistance of $1 \times 10^{-2} \Omega \mathrm{m}^{2}$. For the simulations with high inter-layer resistance it becomes clear that the total resistance barely changes going from 10 to 12 layers. On the other hand for the isotropic simulation, adding material in this range still significantly lowers the total resistance. The experimental curve lies somewhere in between these two scenarios, indicating the presence of inter-layer resistance. The FEM simulations do not take into account contact resistance and parasitic resistance of the set-up. For low numbers of layers the measurements and simulations do not fit well.

\section{Discussion AND CONCLUSIONS}

The proposed novel measurement method clearly shows the change in resistance per printed layer. The measurements show strong qualitative similarity for different print settings and materials, enabling an easy comparison of results. Resistance (changes) during measurements are highly correlated with the resistances after cooling down, allowing for optimisation of electrical conductivity in 3D printed conductors and sensors. Since the method uses embedded electrical contacts, these can be used after fabrication as connections to the sensors, and vice versa sensor connections can already be used during fabrication for monitoring.

Several remarks can be made about the results. The measurement results show an interesting difference in height and sharpness of the resistance peak for the two different infill angles in figures 3 and 4 . This can likely be explained from the temperature dependence of the resistivity in combination

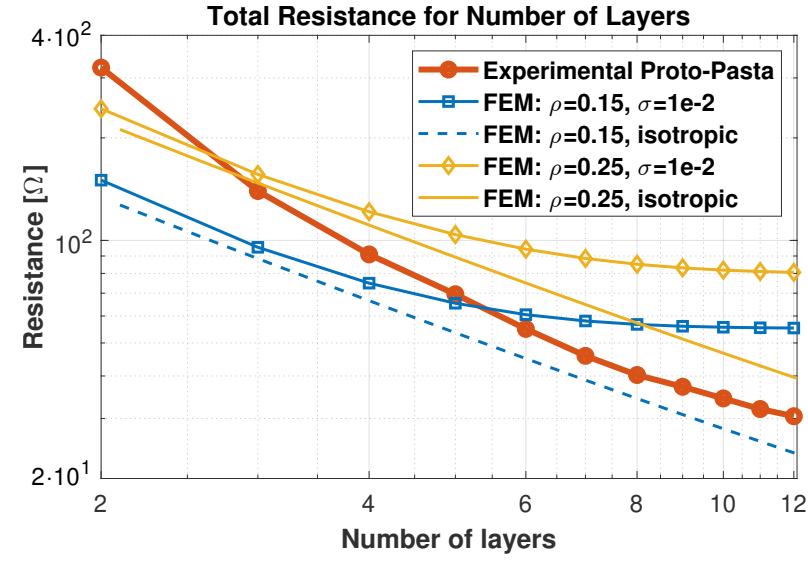

Fig. 5. The total measured resistance after cooling down (extrusion multiplier: $100 \%$, infill angle: $0^{\circ}$ ) compared to FEM simulation results as a function of number of layers. FEM simulations are performed for isotropic conditions (no inter-layer resistance), and high inter-layer resistance of $1 \times 10^{-2} \Omega \mathrm{m}^{2}$.

with the infill angle. For an angle of $0^{\circ}$ the nozzle heats up the sample in parallel to the electrodes, so the current always flows through a just heated section with a high resistivity. For $90^{\circ}$ the nozzle heats up the sample perpendicular to the electrodes, so the current can always flow through sections that have not been heated yet or have already started cooling. In this way the change in resistance over time is more pronounced for an infill angle of $0^{\circ}$. Another remark is that the measurements and FEM simulations do not fit well for small numbers of layers. One explanation for this could be a poor connection between the electrical contacts and the print at the start, giving a high contact resistance. When the first layers are printed on top of the contacts, the nozzle then pushes on them, melts the plastic and in this way lowers the contact resistance. This would be an explanation for the measured high resistance at low numbers of layers. Furthermore the method currently has two major challenges. A first challenge for the new method is the placement of the electrical contacts. Only after placing the contacts one can start measuring. Therefore contacts have to be on the print bed or in the first layers to monitor the resistance for the largest part of the fabrication process. A second challenge for the new method is posed by the thermal effects. Cooling down of the sample in between printing helps to get a more reliable resistance measurement. However, it is still unclear how the inhomogeneous heating from the bed heating below and the nozzle above influences the resistivity throughout the sample and if cooling down is sufficient. This challenge could be reduced significantly by heating the entire environment around the sample up to the bed temperature.

Future research is aimed at gaining more understanding of the method. A next step will be studying the use of the method for different print parameters as well as electrode and print geometries to see how it can be used for optimisation of 3D-printed electrical conductive structures. Finally it can be researched if the optimised parameters obtained by studying fusion in conductive materials can also be used for improving regular mechanical prints. 


\section{REFERENCES}

[1] A. Dijkshoorn, P. Werkman, M. Welleweerd, G. Wolterink, B. Eijking, J. Delamare, R. Sanders, and G. J. Krijnen, "Embedded sensing: Integrating sensors in 3-D printed structures," Journal of Sensors and Sensor Systems, vol. 7, no. 1, pp. 169-181, 2018.

[2] Y. Xu, X. Wu, X. Guo, B. Kong, M. Zhang, X. Qian, S. Mi, and W. Sun, "The boom in 3d-printed sensor technology," in Sensors (Basel, Switzerland), 2017, pp. 1-37.

[3] M. R. Khosravani and T. Reinicke, "3D-printed sensors: Current progress and future challenges," Sensors and Actuators, A: Physical, vol. 305, p. 111916, 2020. [Online]. Available: https://doi.org/10.1016/j.sna.2020.111916

[4] S. W. Kwok, K. H. H. Goh, Z. D. Tan, S. T. M. Tan, W. W. Tjiu, J. Y. Soh, Z. J. G. Ng, Y. Z. Chan, H. K. Hui, and K. E. J. Goh, "Electrically conductive filament for 3D-printed circuits and sensors," Applied Materials Today, vol. 9, pp. 167-175, 2017.

[5] P. F. Flowers, C. Reyes, S. Ye, M. J. Kim, and B. J. Wiley, "3d printing electronic components and circuits with conductive thermoplastic filament," Additive Manufacturing, vol. 18, pp. 156 - 163, 2017.

[6] A. Joshi, J. K. Goh, and K. E. J. Goh, "Chapter 3 - polymer-based conductive composites for $3 \mathrm{~d}$ and $4 \mathrm{~d}$ printing of electrical circuits," in $3 D$ and $4 D$ Printing of Polymer Nanocomposite Materials, K. K. Sadasivuni, K. Deshmukh, and M. A. Almaadeed, Eds. Elsevier, 2020, pp. $45-83$.

[7] B. Hampel, S. Monshausen, and M. Schilling, "Properties and applications of electrically conductive thermoplastics for additive manufacturing of sensors," Technisches Messen, vol. 84, no. 9, pp. 593-599, 2017.

[8] J. Zhang, B. Yang, F. Fu, F. You, X. Dong, and M. Dai, "Resistivity and Its Anisotropy Characterization of 3D-Printed Acrylonitrile Butadiene Styrene Copolymer (ABS)/Carbon Black (CB) Composites," Applied Sciences, vol. 7, no. 1, p. 20, 2017.

[9] G. Wolterink, R. Sanders, and G. Krijnen, "Thin, Flexible, Capacitive Force Sensors Based on Anisotropy in 3D-Printed Structures," Proceedings of IEEE Sensors, vol. 2018-Octob, pp. 2-5, 2018.

[10] S. Mousavi, D. Howard, F. Zhang, J. Leng, and C. H. Wang, "Direct 3d printing of highly anisotropic, flexible, constriction-resistive sensors for multidirectional proprioception in soft robots," ACS Applied Materials \& Interfaces, 2020, pMID: 32129594.

[11] H. Watschke, K. Hilbig, and T. Vietor, "Design and characterization of electrically conductive structures additively manufactured by material extrusion," Applied Sciences (Switzerland), vol. 9, no. 4, pp. 1-25, 2019.

[12] A. Dijkshoorn, M. Schouten, G. Wolterink, R. Sanders, S. Stramigioli, and G. Krijnen, "Characterizing the electrical properties of anisotropic, 3d-printed conductive sheets for sensor applications," accepted in IEEE Sensors.

[13] L. Li, R. McGuan, P. Kavehpour, and R. Candler, "Precision enhancement of 3d printing via in situ metrology," 2020, pp. 251-260.

[14] C. Liu, A. C. C. Law, D. Roberson, and Z. J. Kong, "Image analysisbased closed loop quality control for additive manufacturing with fused filament fabrication," Journal of Manufacturing Systems, vol. 51, pp. 75 - 86, 2019.

[15] H. Wu, Y. Wang, and Z. Yu, "In situ monitoring of fdm machine condition via acoustic emission," International Journal of Advanced Manufacturing Technology, vol. 84, no. 5-8, pp. 1483-1495, 2016, cited By 43.

[16] Y. Li, W. Zhao, Q. Li, T. Wang, and G. Wang, "In-situ monitoring and diagnosing for fused filament fabrication process based on vibration sensors," Sensors (Switzerland), vol. 19, no. 11, 2019.

[17] C. Kousiatza and D. Karalekas, "In-situ monitoring of strain and temperature distributions during fused deposition modeling process," Materials and Design, vol. 97, pp. 400-406, 2016.

[18] T. J. Coogan and D. O. Kazmer, "In-line rheological monitoring of fused deposition modeling," Journal of Rheology, vol. 63, no. 1, pp. 141-155, 2019.

[19] E. Ferraris, J. Zhang, and B. V. Hooreweder], "Thermography based in-process monitoring of fused filament fabrication of polymeric parts," CIRP Annals, vol. 68, no. 1, pp. 213 - 216, 2019.

[20] S. F. A. Acquah, B. E. Leonhardt, M. S. Nowotarski, J. M. Magi, K. A. Chambliss, T. E. S. Venzel, S. D. Delekar, and L. A. Al-Hariri, "Carbon nanotubes and graphene as additives in 3d printing," in Carbon Nanotubes, M. R. Berber and I. H. Hafez, Eds. Rijeka: IntechOpen, 2016 , ch. 8 .
[21] Y. Liu, H. Zhang, H. Porwal, J. Busfield, T. Peijs, and E. Bilotti, "Pyroresistivity in conductive polymer composites: a perspective on recent advances and new applications," Polymer International, vol. 68, no. 3, pp. 299-305, 2019.

[22] ProtoPlant, makers of Proto-pasta. Composite PLA - Electrically Conductive Graphite. Accessed: 31-01-2017. [Online]. Available: https://www.proto-pasta.com/

[23] Palmiga Innovation. Material info for PI-ETPU 95-250 Carbon Black the conductive and flexible 3D printing filament. Accessed: 05-03-2019. [Online]. Available: http://rubber3dprinting.com/pi-etpu-95-250-carbonblack 